\title{
The Role of Parents' Incarnated Cultural Capital on Their Children's Studying Habit
}

\author{
Mehran Karimian Yousefi' ${ }^{1}$ Rozita Sepehrnia ${ }^{2 *}$, Nassim Majidi Ghahroudi ${ }^{3}$ \\ ${ }^{1}$ Department of Social and Communication Sciences, E-Campus, Islamic Azad University, Tehran, Iran \\ ${ }^{2}$ Department of Social sciences, Roudehen Branch, Islamic Azad University, Roudehen, Iran \\ ${ }^{3}$ Department of Communication Sciences and Cultural Studies, Tehran Central Branch, Islamic Azad University, Tehran, Iran \\ Email: ^Sepehrnia@riau.ac.ir
}

How to cite this paper: Yousefi, M. K., Sepehrnia, R., \& Ghahroudi, N. M. (2018). The Role of Parents' Incarnated Cultural Capital on Their Children's Studying Habit. Advances in Literary Study, 6, 30-40. https://doi.org/10.4236/als.2018.61004

Received: January 5, 2018

Accepted: January 26, 2018

Published: January 29, 2018

Copyright (c) 2018 by authors and Scientific Research Publishing Inc. This work is licensed under the Creative Commons Attribution International License (CC BY 4.0).

http://creativecommons.org/licenses/by/4.0/

(c) (i) Open Access

\begin{abstract}
The purpose of this study, as an applied study, was to investigate the role of parents' incarnated cultural capital on their children's studying habits. To do so, the design of the study was chosen to be a survey study. The population of the current study were 1200 parents of the primary school students living in Tehran zone one. Due to the limitation of the study, the Cochran formula was used and 291 individuals were selected. In this study, random-cluster sampling was used and the questionnaire was distributed among the participants. To collect theoretical information, different sources such as the related books and articles were used. To collect information for the matter of the research hypotheses, a questionnaire was used. In the descriptive statistics, first, the necessary indexes were obtained, and then, the reliability and validity of the questionnaire were computed. Moreover, to see the normalization of the data, Kolmogorov-Smirnov was used. Then after, to test the hypotheses, Pearson coefficient, and regression on SPSS have been used. Based on the obtained information, all of the hypotheses were supported and incarnated cultural capital of the parents and its different aspects had positive effects of the studying habit of the children.
\end{abstract}

\section{Keywords}

Incarnated Cultural Capital, Parents, Children's Studying Habits

\section{Introduction}

The incarnated cultural capital is related to the potential part of an individual which is gradually going to be internalized. The educational level of the parents has a crucial role in this regard. The parents, especially mothers, spend most of 
their time with their children in the first years of their life. The children, moreover, use replication to imitate their parents' characteristics. Given that, the educated parents have a higher knowledge about these issues and they can lead to appropriate behaviors by their children if they appropriated their behaviors. When the studying habits are investigated, one cannot ignore the role of family, school, the children book both regarding quantitative and qualitative aspects, economic and social development, and the scarcity of the libraries. The family as the most important place, it can contribute the formation of the children personality. Children who are growing up in the families with high educational degrees, well-being economic and homes in which using cultural productions become a value can be good study followers. The mean of cultural capital in this study is the psychological parents' tendency to use cultural productions, the extent to use cultural production and the education and the job of their parents. The child who grows up in a family with cultural productions, in which the parents spend some of their time studying, in different meeting they exchange their idea about the family and job issues; this child will be different of the family who have low cultural capital. Accordingly, it can be stated that the act of studying has got a very important role in developing the political awareness of different individuals such as students (Qaderi \& Rezaei, 2014). Repeating studying can increase the creativity of a child and help him to be innovative in other stages of his life. Ignoring studying is due to lack of studying habit in the childhood time. It's said that the role of the parents in growing up their children's studying habit should not be ignored. Cultural capital is one of the main indexes regarding cultural tendencies based on which the cultural and artistic tendencies will develop in the parents and they, in their turn, lead to the developing studying habit of their children (Goldenberg, 2014). Given that, the main question of the current study is that: can the incarnated cultural capital of the parents have effect on the studying habit of their children? In the second part of this research, theoretical foundations about the main variables of the research are presented and the hypotheses are stated at the end of it. In the third part, the research method is expressed and then the findings of the hypothesis test are expressed in the fourth section. Finally, the findings of the research have been discussed.

\section{Theoretical Underpinnings and the Hypotheses}

\subsection{Cultural Capital}

The cultural capital is the combination of non-economic elements such as family, social status, different investment and educational commitment, which has a significant role in the academic achievement. From the perspective of Bordio, cultural capital can have three configurations (Bourdieu, 1983): 1) incarnated, meaning that it is in the form of mental and physical characteristics; 2) objected, meaning that it is related to cultural productions (images, books, dictionaries, and machines) which is the trace of the theoretical underpinnings; and 3) institunalized, meaning that it is separated from the objected one since their charac- 
ters are different and they deal with each other in this regard (Kim, 2017).

According to Bordio, in each and every society, there are four exchanging capitals, including economic, social, cultural, and symbolic. The cultural capital means the capacity of an individual to use the cultural production which is internalized during the process of socialization (Dumais, 2015). From the point of view of Bordio, cultural capital includes different habits and tendencies such as knowledge, language, taste, and life style. The key conceptual in this process is habitual action according to Bordio. These habitual actions include the differences in the good and bad tastes and high and low cultures. In this process the children of the high class understand to learn their own class and consider the low class something ignoble. Moreover, the middle class parents try to push their children to read and watch good books and TV programs, respectively. The children of the middle class have the tendency to visit art festival, historical monuments, and theater. Cultural capital is continuously regenerate itself. That said, although people are continuously equipped with new cultural productions during their life, these selections are due to the cultural aspects which are in line with their tastes and attitudes. Hence, they learn the things that are in line with their habits and attitudes and not many changes are seen in this regard (Andres \& Chapain, 2013).

\subsection{Incarnated Cultural Capital}

All the features of cultural capital can be stated in a sentence that this capital is in essence related to incarnation and incarnation is a prerequisite for it. The accumulation of cultural capital in a state of incarnation, in the form of what is called culture and education and treatment, is already in the process of becoming part of the incarnation. The desired incarnation is like muscle building or tanning, which is impossible to perform in the second form (so that any deleterious effects are excluded). The action of getting and acquiring are related to the self. The incarnated cultural capital is a part of the human so it is not easy to convey it through giving present or by buying books. This capital will be manifested when the individual is mentally and physically ready. This capital has got a potential feature which can be gradually instructed and with passing the time, it will be internalized and it is not easy to convey it on the spot (Parsons \& Carlone, 2013).

\subsection{Parents' Cultural Capital: Studying and Academic Achievement of the Children}

According to Bordio's cultural capital theory different organizations of the society have role in regenerating. The educational system is not that much equal regarding different thing as stated by the individuals of the high class society. As people in different social class are with different norms, it is the dominant social class which can set their norms as the standard norms of the society. The educational system has got the assumptions of such norms, but very few people of the 
high social class learn them in their families. Indeed, the responsibility of the educational system and schools is to internalize the norms in the people of a society. That said, the culture and language of the schools are more in line with that of the high social class and the students from this social class have a pretty good knowledge of such norms compared to other students. The students of the lower social class need to first eradicate their own culture and then learn the new one or they drop out. According to the theory of cultural stimulation the academic achievement is a motive of development for the lower social class; meaning that the people of lower social class will regard the higher social class norms as appropriate things, so they try to achieve them by studying. Consequently, success and academic achievement are of great interest to them. Hence, their educational sensitivity, that is, the amount of attention paid by parents to the education of their children-the mental dimension of engagement in the curriculum-as well as the awareness and follow up of their children's educational affairs-the practical dimension of engagement in the curriculum, similarly, anxiety about educational failure and its negative consequences for this class are very highlighted (Bourdieu, 1983). Considering the foundations for achieving the main goal of the research, the following hypotheses are considered and tested:

\subsubsection{Main Hypothesis}

The incarnated cultural capital of the parents has effect of the studying habit of their children.

\subsubsection{Sub-Hypothesis}

1) The economic status of the parents has a statistically significant relationship to the studying habit of their children.

2) The social status of the parents has a statistically significant relationship to the studying habit of their children.

3) There is a statistical significant relationship between the skill role of parents and the studying habit of their children.

4) There is a statistical significant relationship between the professional role of parents and the studying habit of their children.

5) There is a statistical significant relationship between the educational role of parents and the studying habit of their children.

\section{Methodology}

The current study was a descriptive-quantitative one which can be regarded as a survey study due to its data collection procedure. The population of the current study was 1200 parents of the primary school students of the first zone of Tehran. In this research, the Cochran formula and Morgan table are used to sample the sample size. According to the type of present research that we are not aware of the variance of the society and the probability of success or failure of the variable, and we cannot use the statistical formulas to estimate the sample size, we use the Morgan table. This table shows the maximum number of samples. Also, 
in cases where there is no community variance or error percentage, we can use the famous Morgan table to estimate sample size. When the error is set to 0.05 and the volume of the community is known, we will come to the results of this table. In principle, this table is provided for convenience in determining the sample size with tolerable error (0.05) and in different situations. Regarding the limited number of society through Cochran's formula, 291 samples were selected by Morgan table extraction.

The data collection instrument was a researcher-made questionnaire which had three sections including ethnographic characteristics (age, gender, education, experience), the parents' incarnated cultural capital which was based on Bordio's five criteria. The Cronbach Alpha was used to determine the reliability due to its general availability and greater use. In this method, the components or parts of the test are used to measure the reliability. A sample consisting of 15 questionnaires was pre-tested in two periods and then using the data obtained from these questionnaires through SPSS software to calculate the confidence coefficient of alpha. Given the fact that the alpha coefficient obtained was 0.70 , which was an acceptable index, it was revealed that the questionnaire had the appropriate reliability for measuring all the hypotheses. With regard to the Cronbach's coefficient, which is more than 0.7 , it can be concluded that each of the subscales of the questionnaire has an appropriate reliability.

The content validity of this questionnaire was approved by 15 experts of the field (Appendix 1). In the review of face validity, after the distribution of the questionnaire, some typographical errors and wording of the questions were corrected, some questions were separated, some were questions deleted; however, there was no particular change in the layout of the questions. Out of 15 distributed questionnaires, 15 questionnaires were returned and confirmed. The approximate time of response to the questionnaire was estimated to be 10 to 15 minutes. To determine the normality of the data and the type of appropriate tests for their analysis, the Kal-Mograzmarnoff test was used. It should be noted that SPSS was used to analyze the data. To verify the correlation between the variables and each of the dimensions of the model, Pearson correlation coefficient and regression were used.

\section{Results}

Based on the descriptive analyses, it was revealed that the most frequency, about $87.5 \%$, belonged to the males as the respondents of the questionnaire in the age range of 40 - 50. Regarding educational level, the most frequent was BA holders and about $57.5 \%$ of the respondents had 10 - 15 years of experience.

\subsection{Data Normalization}

The Kolmogorov-Smirnov test was used to check the normal distribution of data. This test, in one-sample mode, compares the observed cumulative distribution function with the expected cumulative distribution function in a variable at 
the sequential measurement level. In other words, in this test, the distribution of an attribute in a sample with a distribution for which the population is supposed to compare. In interpreting the test results, if the level of significance (sig) is greater than the probability level of error $(\alpha=0.05)$, then the desired distribution is normal. However, if the sig value is smaller than the probability level of the error $(\alpha=0.05)$, then the distribution will not be super-normal.

Based on the information obtained from Table 1, since the amount of Kolmogorov-Smirnov is less than the $p$-value it can be inferred that the expected distribution and the observed distribution for all the variables are statistically significant and so the distribution is normal. Given that, the parametric statistics can be used.

\subsection{Hypotheses Testing}

\section{Main Hypothesis}

Based on these coefficients in Table 2, one can first explain the variable regression of the children's habit to the study based on the independent variables and the fixed coefficient value, and secondly, it can be predicted the value of the effect of each independent variable for one unit of change in the dependent variable. Therefore, the main hypothesis of the research is confirmed.

\section{The first sub-hypothesis}

In Table 3 based on F and $p$-value (Sig. $<0.05$ ) it can be concluded that there is a statistically significant relationship between the parents' economic status and

Table 1 . The normality of data distribution by Kolmogorov-Smirnov test.

\begin{tabular}{cccccc}
\hline & Kolmogorov & Sig & $p$-value & Results \\
\hline Parents' economic status & 1.409 & 0.320 & 0.05 & Normal \\
Parents' social status & 1.220 & 0.441 & 0.05 & Normal \\
Parents' skill role & 1.303 & 0.288 & 0.05 & Normal \\
Parents' professional role & 1.559 & 0.355 & 0.05 & Normal \\
Parents' educational role & 1.427 & 0.276 & 0.05 & Normal \\
Parents' incarnated cultural capital & 1.559 & 0.384 & 0.05 & Normal \\
Studying habit & 1.776 & 0.547 & 0.05 & Normal
\end{tabular}

Table 2. Regression values.

\begin{tabular}{ccccc}
\hline Model elements & Non-standard B & Standard B & $p$-value & Sig \\
\hline Fix value & 1.887 & - & 5.766 & 0000 \\
Parents' economic status & 0.162 & 0.203 & 4.582 & 0.008 \\
Parents' social status & 0.196 & 0.246 & 3.920 & 0.019 \\
Parents' skill role & 0.172 & 0.291 & 2.043 & 0.041 \\
Parents' professional role & 0.201 & 0.338 & 5.998 & 0.033 \\
Parents' educational role & 0.299 & 0.455 & 6.113 & 0.007 \\
\hline
\end{tabular}


Table 3. Two-variable regression.

\begin{tabular}{cccccc}
\hline & Durbin Watson & R2 & F & $p$-Value & Beta \\
\hline $\begin{array}{c}\text { Parents' economic status and their } \\
\text { children's studying habit }\end{array}$ & 1.764 & 0.166 & 18.384 & 0.000 & 0.408 \\
\hline
\end{tabular}

their children's studying habit. Based on beta score it is revealed that the relationship is about 0.408 . Therefore, the first sub-hypothesis of the research is confirmed.

\section{The second sub-hypothesis}

In Table 4 based on $\mathrm{F}$ and $p$-value (Sig. $<0.05)$ it can be concluded that there is a statistically significant relationship between the parents' social status and their children's studying habit. Based on beta score it is revealed that the relationship is about 0.54 . Therefore, this hypothesis is confirmed.

\section{Third sub-hypothesis}

In Table 5 based on F and $p$-value (Sig. $<0.05$ ) it can be concluded that there is a statistically significant relationship between the parents' skill role and their children's studying habit. Based on beta score it is revealed that the relationship is about 0.41 . Therefore, this hypothesis is confirmed.

\section{Forth sub-hypothesis}

Based on F and p-value (Sig. < 0.05) in Table 6, it can be concluded that there is a statistically significant relationship between the parents' professional role and their children's studying habit. Based on beta score it is revealed that the relationship is about 0.48 . Therefore, this hypothesis is also confirmed.

\section{Fifth sub-hypothesis}

Based on F and $p$-value (Sig. $<0.05$ ) in Table 7, it can be concluded that there is a statistically significant relationship between the parents' educational role and their children's studying habit. Based on beta score, it is revealed that the relationship is about 0.50 . Therefore, this hypothesis is also confirmed.

\section{Discussion}

Along with the overall conclusion of the present study, Dani and TaghiPanahi (2008) which investigated the motivation of the primary and guidance schools' students to study. Dani and TaghiPanahi found out that all of the participants had the motivation to study but with different ranks. "Competition", "importance", "pleasure" and "score "were the motives that were higher in the investigated students compared to the others. Moreover, the motivation for avoiding the study included studying for social reasons, studying with a challenging purpose, and the assignment which received less scores than the rest of the motives (Dani \& TaghiPanahi, 2008). The results of the first sub-hypothesis showed that there is a relationship between the economic status of parents and their children's studying habit. Beta has shown that there was a statistically significant relationship between the economic status of parents and their children's studying habit. The results are in line with the mixed method study of Kim (2017) which investigated the role of social and economic status and found out that both of 
Table 4. Two-variable regression.

\begin{tabular}{cccccc}
\hline & Durbin Watson & R2 & F & $p$-Value & Beta \\
\hline $\begin{array}{c}\text { Parents' social status and their } \\
\text { children's studying habit }\end{array}$ & 1.689 & 0.299 & 21.517 & 0.000 & 0.547 \\
\hline
\end{tabular}

Table 5. Two-variable regression.

\begin{tabular}{cccccc}
\hline & Durbin Watson & R2 & F & $p$-Value & Beta \\
\hline $\begin{array}{c}\text { Parents' skill role and their children's } \\
\text { studying habit }\end{array}$ & 1.761 & 0.168 & 12.099 & 0.000 & 0.411 \\
\hline
\end{tabular}

Table 6. Two-variable regression.

\begin{tabular}{cccccc}
\hline & Durbin Watson & $\mathrm{R} 2$ & $\mathrm{~F}$ & $p$-Value & Beta \\
\hline $\begin{array}{c}\text { Parents' professional role and their } \\
\text { children's studying habit }\end{array}$ & 1.779 & 0.236 & 18.115 & 0.000 & 0.486 \\
\hline
\end{tabular}

Table 7. Two-variable regression

\begin{tabular}{cccccc}
\hline & Durbin Watson & R2 & F & $p$-Value & Beta \\
\hline $\begin{array}{c}\text { Parents' educational role and their } \\
\text { children's studying habit }\end{array}$ & 1.801 & 0.256 & 19.339 & 0.000 & 0.506 \\
\hline
\end{tabular}

the factors have role in the academic achievement of the students. Thus, the more the extent and the quality of such factors in a family, the more the academic achievements of their children may be (Kim, 2017). Based on the information obtained regarding the second sub-hypothesis the social status of the parents and their children's studying habit have relationship. Beta has shown that there is a statistically significant relationship in this regard. The results are in line with that of Dumais (2015) in which Dumais stated that cultural capital can develop cultural abilities of individuals such as perseverance, being a student, using computers at home, and so on. It is, moreover, can have an effective and positive relationship with adolescent education (Dumais, 2015).

The results of testing the third sub-hypothesis showed that there was a relationship between parents' skill role and children's studying habit. Beta has shown that there was a statistically significant relationship between parents' skill role and children's studying habit. The results are in line with that of Hamani and AshrafiRazi (2013) which investigate the methods of promoting culture and studying. Hamani and AshrafiRazi stated that the family has got a crucial role in making habits of studying in the children starting from the very birth. The simple poems will set on the mind of child which provide security sense to him and let him try his firs communication with the society. Telling story can provide pleasuring time for the child and be part of the legend of country. Telling stories is another way of familiarizing children with reading pictures before they enter school and creating shared experiences between them and adults. Moreover, one of the most important social consequences of telling story is to bring family members closer together (Hamani \& AshrafiRazi, 2013). 
The results of testing the forth sub-hypothesis revealed that there is a relationship between the professional role of the parents and the studying habits of their children. The beta showed that there is statistically significant a relationship between the professional role of the parents and the studying habits of their children. The results are in line with the results of the study conducted by Haghighatian (2014) in which the researcher studied the relationship between parents' cultural capital and their children's academic achievement in Isfahan. Haghighatian stated that cultural capital has got a very important stance in the recent years and it is related to sustainable capacities and qualities, being preserved, studious, using computer at home, so on. However, adolescent education has always been one of the major issues of sociology. In this study, the relationship between parents' cultural capital and their children educational achievement has been studied. The results of statistical tests showed that parent cultural capital only had a negligible but statistically significant relation to the academic achievement of guidance school students. There was no significant difference between girls and boys in terms of exploitation of cultural capital of parents (Haghighatian, 2014). In the fifth sub-hypothesis, it was determined that the role of parents' educational role with the children's habit had a significant relationship. The beta also indicates that there was a statistically significant relationship between the parents' educational role and their children's studying habit. The results are in line with that of Gracia (2015) in parent-child leisure activities and cultural capital in the UK which investigated the relationship between education and the social class. Based on that, the way parents and their children spend their leisure time is a type of cultural capital. From one perspective, the social class will affect the cultural capital and these factors affect the children's tendency to their academic affairs (Gracia, 2015).

\section{Conclusion}

In a comprehensive analysis, the role of incarnated cultural capital of parents and its related components have been investigated and their roles on the studying habits of the children have been shown. It is suggested to the parents that they consider second language learning and entertainment for their children along going theater important. Moreover, it is suggested that parents select profession which bring them appropriate social class and continue their higher education to grow up their children better. Parents should invest on cultural issues such as to familiarize the children with theater, etc. The possibility of studying will be provided for the children and from the very childhood, parents read books for them to unconsciously push them to study.

\section{References}

Andres, L., \& Chapain, C. (2013). The Integration of Cultural and Creative Industries into Local and Regional Development Strategies in Birmingham and Marseille: Towards an Inclusive and Collaborative Governance? Regional Studies, 47, 161-182.

https://doi.org/10.1080/00343404.2011.644531 
Bourdieu, P. (1983). The Field of Cultural Production, or: The Economic World Reversed. Poetics, 12, 311-356. https://doi.org/10.1016/0304-422X(83)90012-8

Dani, M. H., \& TaghiPanahi, F. (2008). Study of Factors Affecting Study Motivation in Primary and Secondary School Students. Journal of Library and Information Science, 43, 38-20.

Dumais, S. (2015). Cultural Capital and Education. International Encyclopedia of the Social \& Behavioral Sciences (Second Edition), 2015, 375-381. https://doi.org/10.1016/B978-0-08-097086-8.10433-7

Goldenberg, B. M. (2014). White Teachers in Urban Classrooms: Embracing Non-White Students' Cultural Capital for Better Teaching and Learning. Urban Education, 49, 111-144. https://doi.org/10.1177/0042085912472510

Gracia, P. (2015). Parent-child Leisure Activities and Cultural Capital in the United Kingdom: The Gendered Effects of Education and Social Class. Social Science Research, 52, 290-302. https://doi.org/10.1016/j.ssresearch.2015.02.005

Haghighatian, M. (2014). Cultural Capital and Intergenerational Reproduction: The Impact of Parent's Cultural Capital on the Student's Achievement in Isfahan City. Intercultural Studies, 9, 53-70

Hamani, M., \& AshrafiRazi, H. (2013). Investigating Strategies for Promoting the Culture of Reading and Reading. Islamic Republic of Iran Scientific-Professional Monthly Journal, 11, 54-42.

Kim, S. (2017). A Longitudinal Mixed Methods Study of Parents' Socioeconomic Status and Children's Educational Attainment in Dalian City, China. International Journal of Educational Development, 52, 111-121. https://doi.org/10.1016/j.ijedudev.2016.10.007

Parsons, E. C., \& Carlone, H. B. (2013). Culture and Science Education in the 21st Century: Extending and Making the Cultural Box More Inclusive. Journal of Research in Science Teaching, 50, 1-11. https://doi.org/10.1002/tea.21068

Qaderi, T., \& Rezaei, S. (2014). The Relationship between Cultural Capital and Youth Trend in Fashion (Difference between Girls and Sons). Journal of Social Sciences, 21, 1-35. 


\title{
Appendix 1
}

The Names of 15 Experts for Approved the Content Validity of the Questionnaire

\author{
Ali Mehregan \\ Amir Ghodsi \\ Sepideh Tarzaminejad \\ Nasser Asadi \\ Molouk Seyyed Abadi \\ Nikou Sarkhosh \\ Gita Ali Abadi \\ Naeema Tolouei Ashleghi \\ Omid Jahanshahi Fard \\ Ali Akbar NonahalNahar \\ Seyyed Mehdi Mirbod \\ Manouchehr Makki \\ Kamran Ganji \\ Rozita Sepehrnia \\ Nasim Majidi Ghahroudi
}

